\section{Competitividad de la \\ agroempresa en un mundo globalizado}

\author{
Óscar Corrales González*
}

\section{Resumen}

La competitividad es un elemento de gran importancia en la época contemporánea sobre todo cuando las barreras comerciales vienen desapareciendo y la apertura de los mercados hace que el mundo sea un solo país" comercialmente hablando. Se requieran elementos diferenciantes que consoliden las ventajas competitivas alcanzadas y que se vuelvan sostenibles en el tiempo, mediante mecanismos generados a lo interno de las empresas así como por los aportes que los gobiernos hayan incorporado en sus politicas y que benefician directamente el entorno social y económico, con lo que se logra una mayor atracción de inversio-

* Ingeniero Agrónomo de la Universidad de Costa Rica, con un posgrado en Administración de Empresas con énfasis en Mercadeo. Actualmente es profesor de la UNED.

* Este trabajo es parte de una Conferencia impartida en la Universidad Estatal a Distancia el 15 de marzo del año 2006.

Rec. 14-12-05 Acep. 28-03-06 nes y desde el punto de vista ambiental, un desarrollo sustentable y amigable con la naturaleza. En Costa Rica se requieren implementar algunos elementos para lograr un mayor desarrollo, como el fortalecimiento de las ventajas competitivas dinámicas, reducción de la baja productividad y dependencia de la mano de obra barata y malas prácticas laborales, mejora en el desempeño de las negociaciones multilaterales actuales y futuras, desarrollo de un clima de negocios mas propicio para la inversión, el impulso de los negocios con visión de cluster o encadenamientos, incorporar mayor valor agregado a la producción y controlar la explotación no sustentable de los recursos naturales con degradación del medio ambiente.

\section{Palabras clave}

Competitividad, ventajas competitivas, calidad, productividad, clusters y valor agregado.

\section{Abstract}

Nowadays, competitiveness is an important element since commercial boundaries are disappearing and the opening of markets, commercially speaking, causes "the world to be one country." Differential elements are required, particularly those strengthening already-enjoyed and timesustainable competitive advantages through mechanisms generated internally in the enterprises and contributions included in governmental policies benefiting social and economic environments directly; important investments will then be attracted and, from the environmental perspective, a sustainable and envi- 
ronmental-friendly development will be ensured. Some aspects must be taken into account in Costa Rica in order to achieve a better development. The following can be mentioned among them: to strengthen dynamic competitive advantages, reduce low productivity, cheap-labor dependence and the number of labor malpractices, improve current and future multilateral negotiation performances; foster an investment-favorable business environment and commercial dealings with vision for clusters, incorporate a higher added value to production and to control non-sustainable exploitation of natural resources harming the environment.

\section{Key words}

Competitiveness, competitive advantages, quality, productivity, clusters and added value.

\section{Introducción}

Los diferentes esquemas de desarrollo socioeconómico, que se han puesto en práctica en Costa Rica, han buscado poner en evidencia las ventajas comparativas y competitivas alcanzadas, que permitan aumentar su participación efectiva en los mercados internacionales.

Por lo anterior los diferentes sectores de la economía laboral, y en el proceso sistemático moderno de apertura de las economías, donde se presenta una fuerte integración de los mercados mundiales, enfren- tan día a día una competencia más agresiva, tecnificada e informada, por lo que en este sentido es fundamental revisar y poner en práctica estrategias que les permita mantenerse en el mercado y entender aquellas otras puestas en práctica por empresas exitosas y comprender la denominada revolución de mercadeo y ventas.

Sin lugar a dudas las gerencias generales de mercadeo y los diferentes equipos de trabajo en las empresas deben contar, entre otras cosas de las nuevas herramientas que les ayuden a fortalecer los principales conceptos utilizados hasta ahora, no solo para participar activamente en el mercado nacional sino en mercados regionales, extraregionales o mundiales, para lograr adaptarse a los cambios tan rápidos que se presentan más frecuentemente y en forma imprevista; de igual forma, deben incorporar normas de calidad, información, mecanismos de intercambio internacional, entre otros, como ventajas competitivas y diferenciadoras, que sean el mecanismo para lograr un mayor posicionamiento $\mathrm{y}$, por ende, un incremento en la rentabilidad por la venta de los productos o servicios que se ofrezcan y sobre 
todo un crecimiento en general de la empresa y de la economía del país.

En el campo de la economía rural, tipificada esencialmente en el marco de la agroempresa, un elemento esencial que debe conseguirse sin lugar a dudas por la importancia que reviste en esta época, es lograr el equilibrio socio- económico, que permita el desarrollo en armonía con el medio ambiente y con lo político institucional (sustentable y amigable), que dignifique al productor, de tal forma que se vea estimulado a continuar produciendo (alimento o servicios) para la población nacional e internacional según sea el caso.

\section{La competitividad}

La especie que sobrevive no es la más fuerte, ni la más inteligente, sino aquella que mejor se adapta al cambio. La globalización, la microtecnología, la revolución genética que se anuncia, la instantánea y casi gratuita comunicación internacional, han creado una dinámica tan revolutiva y cambiante del entorno que la empresa postmoderna se ve obligada a una adaptación y transformación continua. (Brenes, 2002:2).
Con base en lo anterior, el éxito de las empresas en la sociedad contemporánea repleta de comunicación, se ve mejorado cuando se logra crear una posición en la mente de los clientes potenciales, una posición que considere no solo las propias fortalezas y debilidades de cada compañía sino también la de los clientes (Lovelock 2004:5).

En un mundo globalizado, donde las megatendencias y los megamercados son los nuevos elementos del quehacer empresarial, se pone de manifiesto la imperiosa necesidad de la competitividad y esta puede ser definida como la capacidad de una empresa o persona para mantener en forma sistemática las ventajas comparativas, que le permitan alcanzar, mantener y mejorar una determinada posición en el entorno socioeconómico donde se desenvuelve, en equilibrio con el medio ambiente.

La competitividad en un Mercado Local puede ser representada como se muestra en la figura 1.

En este caso específico se pueden determinar todas las interrelaciones que se presentan a lo largo del mercado, desde el productor, el canal de comercialización utilizado y el consumidor intermedio o final. 
Figura 1

Representación de la competitividad en un mercado local
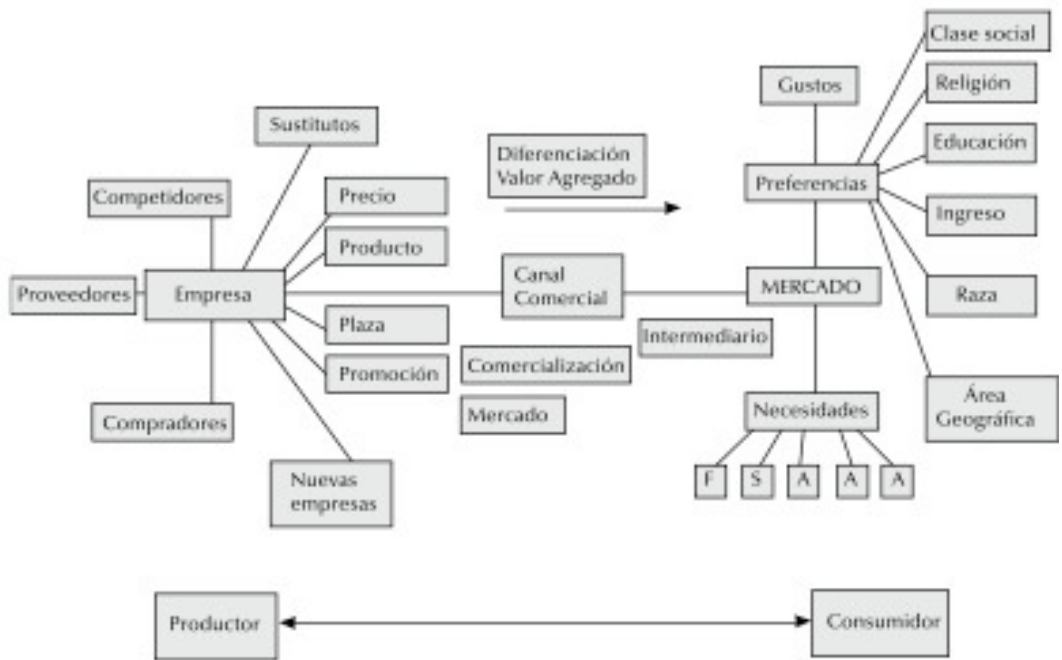

Figura 2

Competitividad en un mercado globalizado

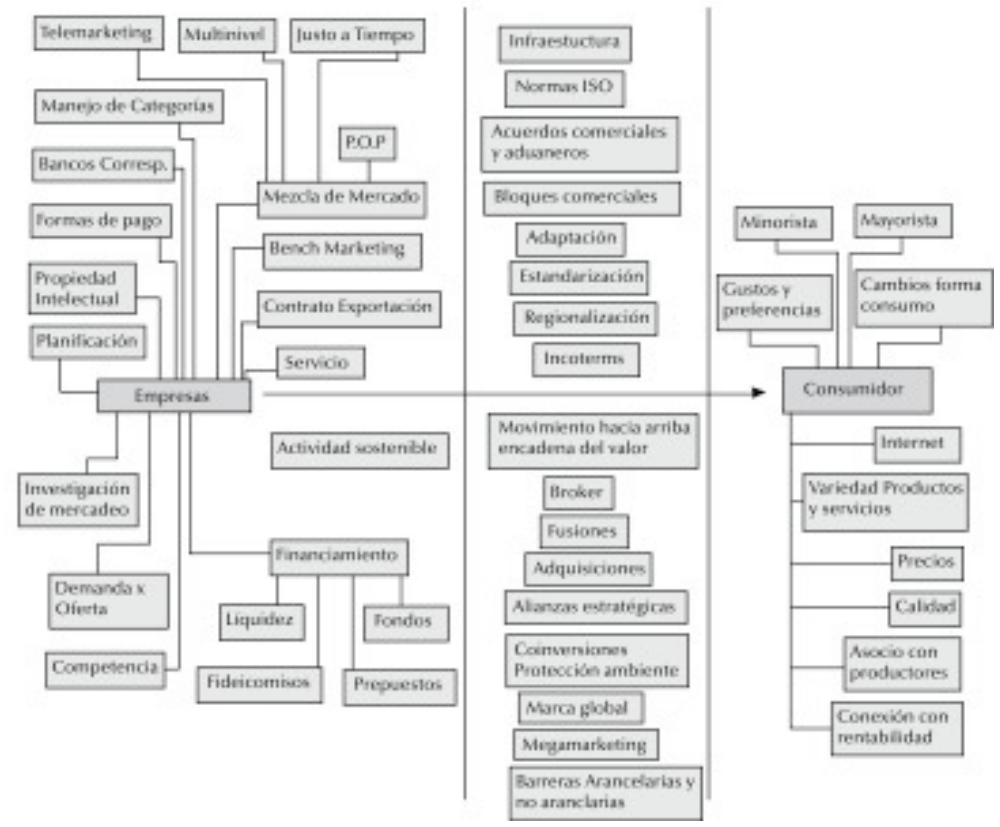


En la figura 2, se muestra la representación gráfica de la competitividad que se presenta en un mercado globalizado, saber:

Con base en lo anterior es importante indicar que:

1. se presentan mayores actividades $y$ requerimientos que permiten mantener la posición competitiva cuando "todo el mundo" se vuelve un solo país.

2. se hacen uso de nuevos esquemas de negocios como las alianzas estratégicas, fusiones, adquisiciones, megamarketing, entre otras.

3. la exigencia de nuevas competencias laborales en el campo profesional se acentúa debido al aumento de interrelaciones, entre ellas el manejo de otros idiomas y el conocimiento de las diferencias culturales que se presentan.

4. participación del profesional en diferentes campos del saber (polifuncionalidad).

5. incorporación de la tecnología informática a las labores, utilización de nuevos esquemas de entrega de productos y servicios (campo virtual y e-commerce).
6. requerimiento de mayor flexibilidad en los programas y negociaciones.

7. desarrollo de la investigación constante para determinar los cambios en el entorno y la implementación de los ajustes necesarios para mantenerse competitivo.

8. desarrollo de elementos profesionales complementarios como la creatividad, el liderazgo, la inteligencia emocional y la empatía, entre otros.

\section{Indicadores para medir el nivel de competitividad}

El índice de competitividad actual puede ser determinado mediante el uso del índice macroeconómico establecido en dos categorías, a saber:

\section{El grado de sofisticación de la competencia entre firmas}

Determinado por el nivel de conocimiento, el uso de la tecnología, el capital físico existente y las habilidades gerenciales que posean.

En este sentido el Crecimiento empresarial puede ser determinado haciendo uso de los siguientes índices que se detallan a continuación: 
- De creatividad económica (la innovación y la transferencia de tecnología).

- $\quad$ Financiero (la eficiencia del sistema financiero, los niveles de ahorro e inversión).

- De internacionalización (la integración económica con el resto del mundo). (Barahona, 2000).

\section{La calidad del clima de negocios}

en este sentido contempla la calidad de la infraestructura, las habilidades, la tecnología disponible, las reglas y regulaciones y las instituciones que conforman el contexto en que operan las empresas.

En la figura 3 se muestra esquemáticamente la serie de interrelaciones que se presentan en este sentido.

Figura 3

Clima de negocios que se presenta en la Cadena Productiva

Clima de Negocios en la Cadena Productiva

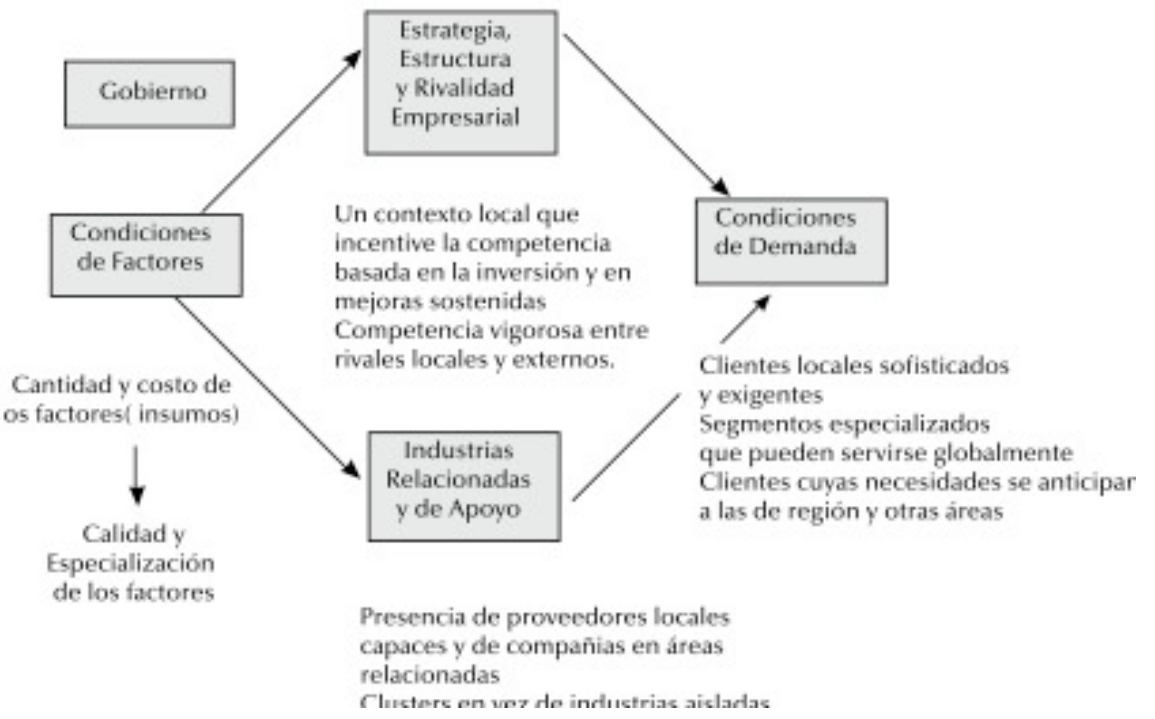

Fuente: Barahona, 2000. 
Uno de los elementos esenciales dentro de este esquema es la importancia que toma el desarrollo de clusters o la unión de empresas relacionadas en lugar de industrias aisladas, como un mecanismo de integración de empresas pequeñas a la cadena productiva, lo que permitirá una ventaja competitiva $\mathrm{y}$ el incremento en información, exigencias y rivalidad entre empresas locales y externas.

\section{La competitividad} costarricense

Según el estudio realizado por Juan Carlos Barahona en el año 2000, se analizaron variables que permitieran conocer cómo nos perciben y cómo nos percibimos, para determinar la posición en que se encuentra el país y establecer de esta forma las políticas y los ajustes necesarios para mejorar la competitividad costarricense. Seguidamente se presentan algunas de las principales variables por considerar en este campo tan importante, a saber:

Cuadro 1

Innovación como elemento competitivo

\section{Innovación}

\begin{tabular}{|l|}
\hline \multicolumn{2}{|l|}{ Ventaja competitiva } \\
\hline \hline $\begin{array}{l}\text { Instituciones de } \\
\text { investigación } \\
\text { científica }\end{array}$ \\
$\begin{array}{l}\text { Desarrollo de } \\
\text { estudios en este } \\
\text { campo }\end{array}$ \\
\hline
\end{tabular}

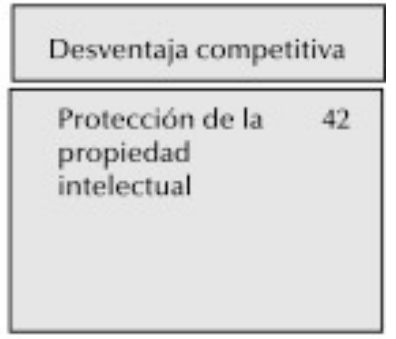

Se está discutiendo en la asamblea legislativa iniciativas relacionadas con propiedad intelectual:

¿Cuándo está cooperando el sector privado a que los diputados entiendan la importancia de este tema para sus actividades? En qué dirección?

¿Qué sigue después?

Fuente: Barahona, 2000.

Espiga 13, Enero-Junio, 2006 149 
Costa Rica cuenta con instituciones reconocidas en este campo; sin embargo se debe avanzar en el desarrollo de un mejor ambiente para la atracción de mayor inversión, mediante elementos que determinen la Protección de la Propiedad Intelectual de las personas físicas y jurídicas.
En este caso es importante mencionar la necesidad de mejorar las condiciones para el desarrollo de un nuevo negocio y el estímulo a la micro, pequeña y mediana empresa, implementando mecanismos como los créditos sin garantía, la atracción y canalización del denominado Capital de Riesgo.

Cuadro 2

Desarrollo de Starups (motores de crecimiento)

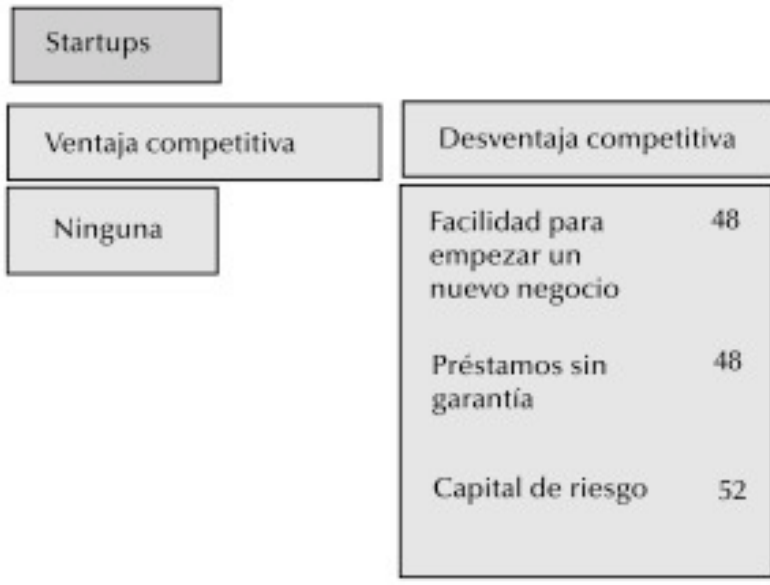

Este es un punto medular para la agenda de desarrollo nacional.

Cuál es el papel de la banca pública y privada?

Fuente: Barahona, 2000. 
A pesar de la fecha en que fue realizado el estudio en mención, todavía hoy se presentan deficiencias en la inversión gubernamental a nivel de infraestructura en caminos, puertos, aeropuertos, telecomunicaciones, electricidad, desarrollo de los ferrocarriles, entre otros, lo que dificulta brindar los requerimientos básicos para la atracción sobre todo de inversiones extranjeras.

Otra de las variables analizadas fue la relacionada con el trabajo desarrollado por los costarricenses; en este sentido, como puede observarse en el cuadro 4, elemen-

Cuadro 3

Participación del Gobierno en actividades que permitan mejorar la competitividad costarricense

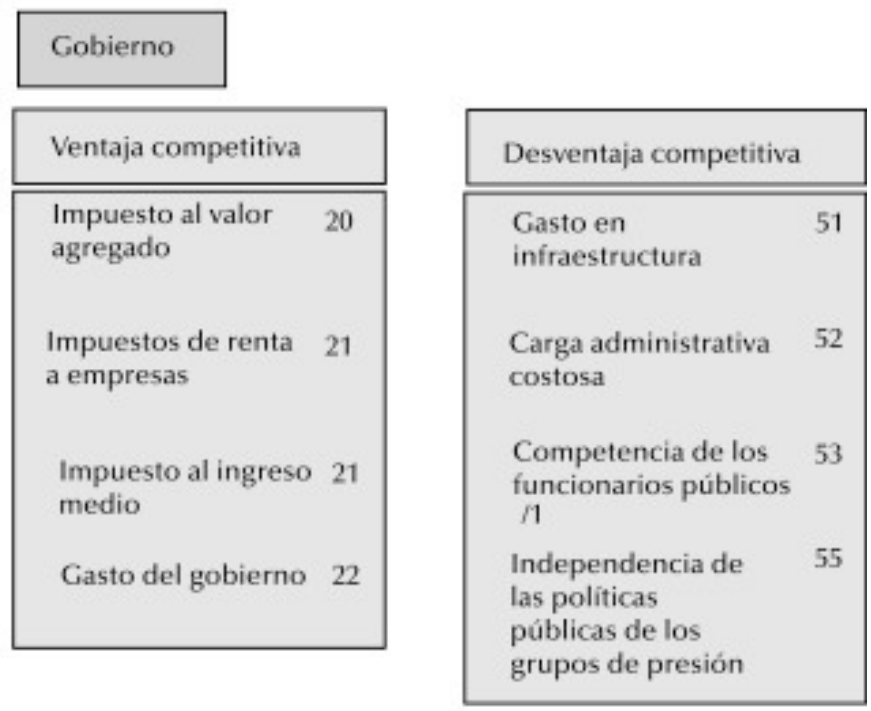

$1 /$ en relación a la competencia del sector privado, En Singapur 4,47 los consideran mejores - CR 2/7

Fuente: Barahona, 2000. 
tos negativos que impiden aumentar la competitividad del país, la dependencia con los grupos de presión a la hora de la negociación de los salarios, la no institución del pago por productividad, y sobre todo la marcada deserción y disminución de la matrícula a nivel de educación secundaria, que afectará en el mediano y largo plazo la generación de nuevos profesionales.

\section{El desafío del desarrollo}

Costa Rica, al igual que el resto de países del mundo, se encuentra inmerso en la toma de una serie de decisiones muy importantes y cruciales que permitirán alcanzar un mayor nivel de intercambio comercial y, por ende, del desarrollo de su aparato productivo y de la sociedad, razón por la cual debe lograr un posicionamiento a nivel nacional, regional y mundial (Antillón 2005:2).

Cuadro 4

Trabajo como elemento de competitividad costarricense

\begin{tabular}{|l|}
\hline \multicolumn{2}{|l|}{ Trabajo } \\
\hline \multicolumn{2}{|l|}{ Ventaja competitiva } \\
\hline $\begin{array}{l}\text { Crecimiento del } \\
\text { empleo }\end{array}$ \\
$\begin{array}{l}\text { Seguros de } \\
\text { desempleo }\end{array}$ \\
$\begin{array}{l}\text { Relaciones } \\
\text { laborales }\end{array}$ \\
$\begin{array}{l}\text { Tasa de } \\
\text { desempleo }\end{array}$ \\
\hline
\end{tabular}

Fuente: Barahona, 2000. 
La concordancia entre las políticas $\mathrm{y}$ acciones gubernamentales como apoyo a los sectores productivos, así como el ajuste de las empresas a los cambios internos necesarios, y sobre todo a aquellos en el entorno externo, será la base para mejorar y consolidar la competitividad, eficiencia y calidad que las identifique en el (los) mercado (s) donde se encuentren brindando sus productos o servicios.
Podría mencionarse una serie de estrategias en este sentido; sin embargo, a criterio del autor, y para los efectos del presente documento, en la figura 4 se presenta esquemáticamente el paso que debería dar el país para ser más competitivo a nivel agroempresarial en un mercado globalizado. En este caso estaría definido en forma general por la transformación de las ventajas comparativas en ventajas competitivas, fortaleciendo aquellas actividades que han logrado alcanzarlas y, por ende, se encuentra totalmente posicionadas en los mercados mundiales.

Figura 4

El desafío del desarrollo

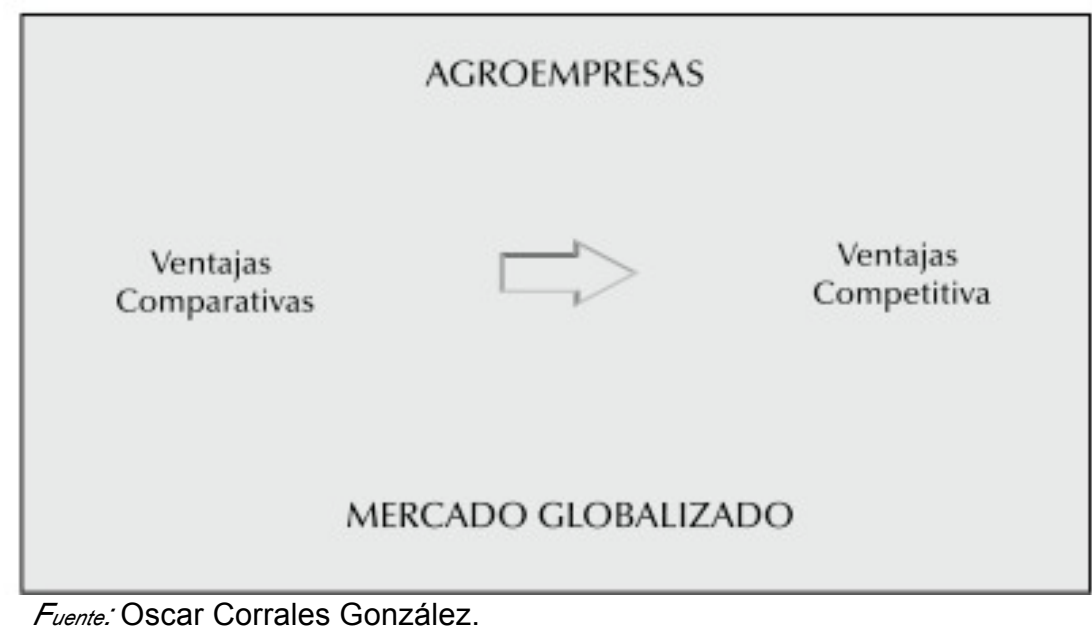

Espiga 13, Enero-Junio, 2006 


\section{Conclusiones}

La competitividad costarricense requiere tomar en cuenta varios elementos para incorporarse totalmente al mercado globalizado que a criterio del autor deben implementarse a la mayor brevedad posible, entre ellos:

1. Como se mencionaba en el punto 4 de este documento, se debe pasar de la dependencia de las ventajas comparativas al fortalecimiento de las ventajas competitivas dinámicas.

2. Dejar atrás la baja productividad, la dependencia de la mano de obra barata y las malas prácticas laborales.

3. Mejorar el desempeño en las negociaciones multilaterales actuales y futuras.

4. Desarrollar un clima de negocios más propicio para la inversión y el comercio en América Latina.

5. Impulsar negocios con visión de clusters (encadenamientos productivos).

6. Encontrar formas de agregar valor a la producción exportable.
7. Controlar la explotación no sustentable de los recursos naturales con degradación del ambiente y la concentración de la actividad económica en centros urbanos (algunos indicadores utilizados son el de cobertura boscosa, recurso hídrico, uso de la tierra, producción de carbono u oxígeno, entre otras).

\section{Bibliografía}

Antillón, Á. et al (2005), El informe de los notables. San José. Editorial EUNED.

Bael, G. (2000), La Exportación está en sus manos. España. Editorial GESTION 2000.

Barahona, Juan C (2000), La Competitividad de las Empresas Costarricenses, Estudio Comparativo. San José. Editorial INCAE.

Brenes, Lizette (2002), Gestión de Comercialización. San José. Editorial EUNED.

Kotler, Philip (2001), Dirección de Marketing. México. Editorial Prentice Hall.

Lovelock, Cristian et al (2004), Administración de Servicios. México. Editorial Prentice Hall.

Núñez, Orlando (2003), La Globalización. www.cipres.org.

Pérez, Luis (2004), El Marketing Social. México. Editorial Prentice Hall. 
Trejos, Alberto y Condo, Arturo (2001), Clima para los negocios en Centroamérica. San José. Editorial INCAE.

Trejos, Alberto y Condo, Arturo (2001), Competitividad Empresarial en Centroamérica. San José. Editorial INCAE.

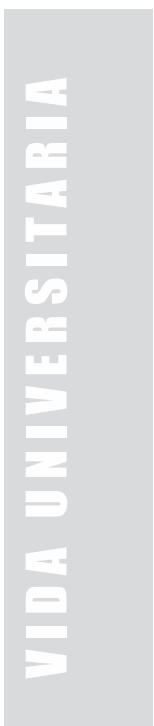


inuestros libros tienen la palabra!
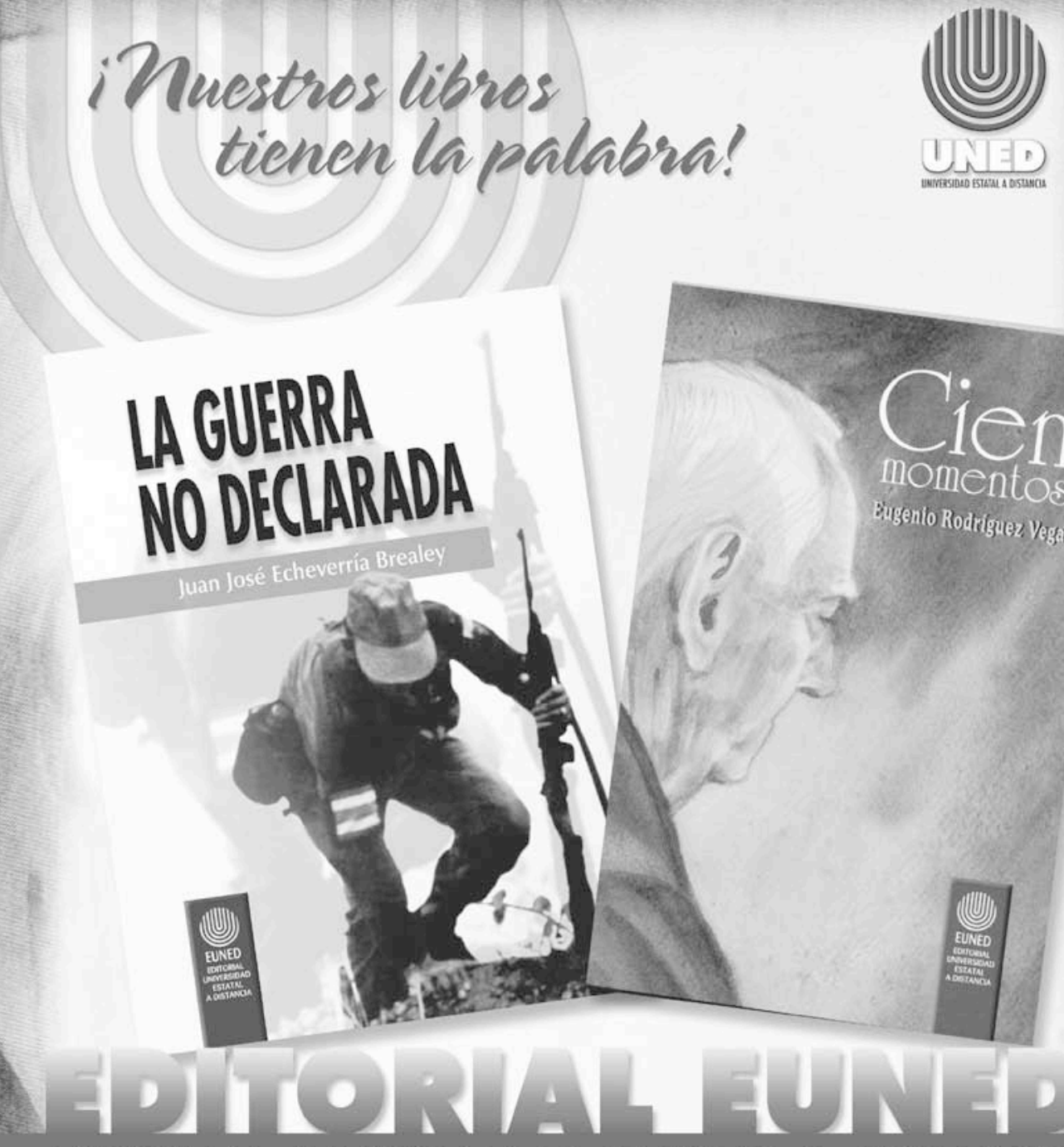

E D I T O I A
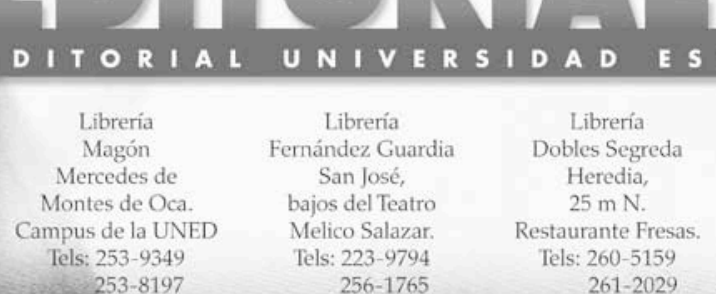
Librería
Carlos Luis Fallas Alajuela,
Frente a Plaza Ferias. Tels: 443-6746 440-4204

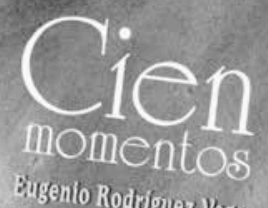

angenio Rodriguez Vega

Tels. 253-9349 256-1765 261-2029

Libreria
Mario Sancho
Cartago,
$200 \mathrm{~m}$ E.
Municipalidad
de Cartago
Tel: $592-1017$

